\title{
Atypical Horner's syndrome: frequency, features and aetiology in a paediatric population
}

\author{
Robert Nutt $^{1} \cdot$ Eibhlin McLoone ${ }^{1} \cdot$ Sonia George ${ }^{1} \cdot$ Michael O'Gallagher $^{1}$
}

Received: 9 September 2019 / Revised: 1 May 2020 / Accepted: 6 May 2020 / Published online: 18 May 2020

(c) The Author(s), under exclusive licence to The Royal College of Ophthalmologists 2020

\begin{abstract}
Background/Objectives Paediatric Horner's syndrome (HS) may present atypically with incomplete or intermittent clinical features, yet could represent sinister pathology including neuroblastoma. We aim to report the frequency and features with which atypical HS presents in our population (Northern Ireland) and to propose an investigation algorithm to aid diagnosis in these challenging cases.

Subjects/Methods Retrospective chart review of all paediatric anisocoria and HS cases presenting to Belfast, Northern Ireland, between 2012 and 2018, identified through searching our paediatric ophthalmology database.

Results Sixty-one eligible cases of anisocoria or HS were analysed. Ten cases of HS were identified, four (40\%) of which presented atypically with incomplete or intermittent features. Two of these four atypical cases were secondary to neuroblastoma. Overall incidence of paediatric HS in Northern Ireland during the study period was at least 2.54 per 100,000.

Conclusions Paediatric HS may present atypically in a significant number of cases. Accordingly, clinicians should consider HS in children with a history of anisocoria or ptosis and have a low threshold for use of pharmacological tests to aid diagnosis.
\end{abstract}

\section{Background}

Paediatric Horner's syndrome (HS) is an important clinical diagnosis which may indicate sinister underlying pathology including neuroblastoma [1, 2]. Furthermore, it can be challenging to diagnose as signs may be subtle, intermittent and deviate from the classical findings of mild ptosis with miosis [3]. Topical apraclonidine $(0.5$ or $1 \%)$, or cocaine (2-10\%) eye drops may be used to help exclude HS in cases of anisocoria, although there is currently no clear consensus regarding which test should be used and when.

Thus far, there are no estimates of what proportion of paediatric HS present with atypical or incomplete features. We aim to (1) estimate the incidence of HS in our population over the study period, (2) establish the number of children with anisocoria who had underlying HS and (3) report the prevalence of atypical HS in this cohort and review the presenting features and aetiology of these cases.

Robert Nutt

Robert.nutt@belfasttrust.hscni.net

1 Royal Victoria Hospital, Belfast Trust, Belfast, UK
In view of our findings, we also propose an investigation algorithm for children presenting with anisocoria and possible HS.

\section{Methods}

Retrospective chart review of children $(<16$ years old $)$ presenting with anisocoria and/or HS between January 2012 (i.e. when our paediatric ophthalmology database was established) to June 2018.

Cases were identified through searching databases of patients who attended the paediatric eye service within the Belfast Trust, for a diagnosis code of "Horner's syndrome", "anisocoria" or "pupil abnormalities" in this time period. Cases were excluded if they were initially evaluated prior to 2012, or when the cause of anisocoria was secondary to a structural cause such as persistent pupillary membrane. Patients' date of birth, age at presentation, clinical features at presentation and subsequent follow up, relevant investigations and outcomes were recorded for all included cases.

HS cases where both ptosis and miosis (where the difference in pupil size was exacerbated by dark conditions) were present on each review were classed as typical. 
HS cases which presented with anisocoria only, ptosis only, or with intermittent signs were classed as atypical.

\section{Results}

Seventy-two cases of anisocoria or HS were identified from our search, 11 of which were excluded because they had initially presented and been diagnosed prior to January 2012. Of the remaining 61 cases, 46 (75.4\%) were diagnosed as physiological anisocoria, 10 (16.4\%) HS, four $(6.5 \%)$ Adie's pupil, one $(1.6 \%)$ pharmacological anisocoria (Fig. 1a).

Of the ten HS cases, six presented "typically" with both ptosis and miosis evident at each review. The remaining four were atypical: two presented with ptosis with later development of miosis; one had intermittent ptosis and later developed intermittent miosis, but examined entirely normally when seen in clinic; one presented with anisocoria only with no ptosis. Two (20\%) of the HS cases were secondary to neuroblastoma, both of which presented with atypical HS. Others were congenital/idiopathic or relating to trauma of some kind (Fig. 1b). The likelihood ratio of having neuroblastoma in those presenting with atypical verses typical HS in this data set is four (that is, a fourfold increased risk of significant pathology in atypical cases).

Considering all 61 cases, 51 presented with just one sign (anisocoria or ptosis) with no history of other signs or symptoms. Of these, in 49 cases no evidence of HS or other significant pathology was identified; the other two were associated with HS but with no apparent underlying cause. The remaining ten cases presented with two of more of the following: ptosis (intermittent or otherwise), anisocoria or presence of significant other history including anisocoria/ ptosis/neck mass/facial flushing. Of these, eight cases were confirmed as HS, two with underlying neuroblastoma. The remaining two of these ten cases were negative for HS on pharmacological testing, although they were consistant with HS clinically. In terms of predictive values, this corresponds to a positive predictive value of $80 \%$ having $\mathrm{HS}$ confirmed with tests in those presenting with two or more features of HS. The negative predictive value for having HS in those presenting with just one feature is $96.1 \%$ based on this data.

\section{Atypical HS cases}

\section{Case 1}

A 12-week-old baby was referred to paediatric ophthalmology with mild left ptosis. The patient had also been seen recently by Ear Nose and Throat (ENT) colleagues who had

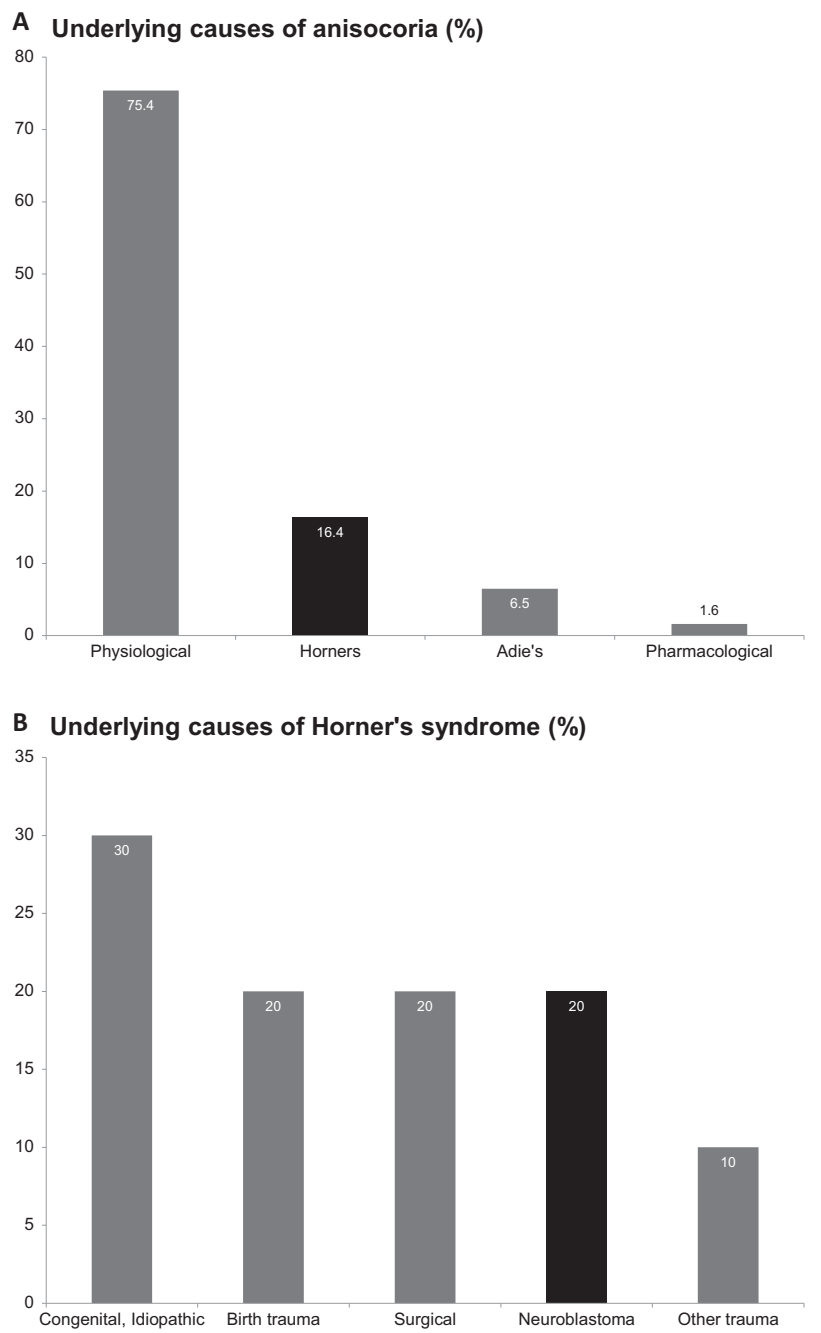

Fig. 1 Relative frequencies and underlying causes of anisocoria (a) and HS (b).

diagnosed a reactive neck lymph node following an episode of tonsillitis. There was no other medical history of note. On initial ophthalmic assessment, mild left ptosis was noted. Subtle anisocoria was also noted in room light with pupils measuring $3 \mathrm{~mm}$ right and $2 \mathrm{~mm}$ left, however in dark conditions the anisocoria resolved with both pupils dilating to $6 \mathrm{~mm}$ (i.e. not in keeping with miosis). Therefore, it was felt the child was unlikely to have HS but rather a congenital ptosis with a degree of physiological anisocoria in certain conditions. Ocular examination was otherwise normal. No pharmacological tests were done as the child was dilated with tropicamide $1 \%$ (following pupil assessment) for fundus examination. A review was planned for 6 weeks' time; however, a week later the child was reviewed by ENT who noted the neck mass had enlarged and arranged ultrasonography (USS) and subsequently computed tomography of the neck, chest abdomen and pelvis (Fig. 2a). This identified a large neck mass and evidence of metastatic spread to the liver. Biopsy showed poorly differentiated 


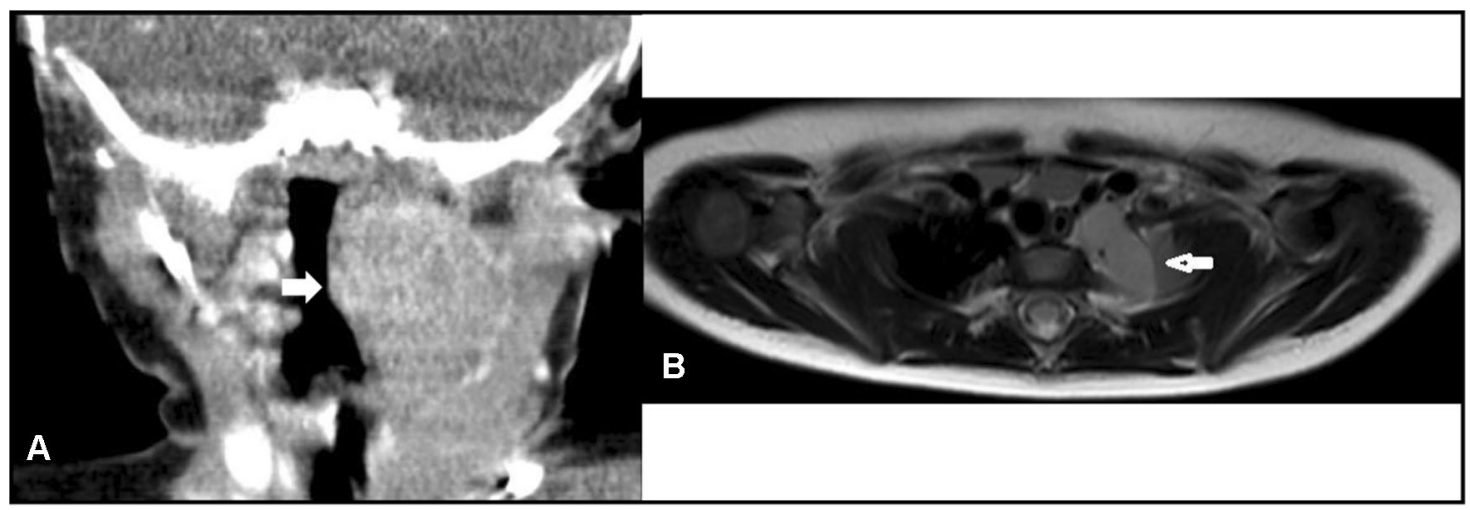

Fig. 2 Radiological images showing neck masses causing atypical Horner's syndrome in two patients. a Case 1, Coronal CT showing left sided neuroblastoma in the neck (arrow). On presentation, the

neuroblastoma. The child responded well to chemo-therapy with regression of both the tumour and metastases. Interestingly, 1 year following the diagnosis, the child had no obvious ptosis but had developed left miosis.

\section{Case 2}

Parents of a 7-month-old girl who had no significant medical history took their child to a walk-in eye casualty having noticed left ptosis on occasions since she was about 4 months old. More recently they had also noticed the pupils were sometimes different sizes. On initial examination there was no ptosis evident, but the left pupil was noted to be 1-mm smaller than the right pupil in light and dark conditions. An initial diagnosis of probable physiological anisocoria was made and review was arranged for 1 week's time by the paediatric ophthalmology team, at which time the child examined normally with no ptosis or anisocoria present. However, the mother had taken photos when the child had signs which showed left ptosis and miosis (Fig. 3a). No pharmacological tests were carried out as the photos alone were felt to be sufficiently concerning for HS, albeit intermittent. Urgent magnetic resonance imaging (MRI) of the head and neck was arranged which showed a neck mass measuring $2 \times 3 \mathrm{~cm}$ (Fig. 2b). Biopsy of the lesion was carried out within a week which confirmed ganglioneuroblastoma. Surgery was deferred whilst awaiting biomarker tests, but in the meantime repeat imaging showed the neuroblastoma was reducing in size. The mass became progressively smaller and is still being observed with scans periodically.

\section{Case 3}

An 8-year-old girl was referred by an optometrist who noted anisocoria which had been present for 3 months according to the father. On examination the pupils measured $3 \mathrm{~mm}$ patient had ptosis but equal pupils dilating to $6 \mathrm{~mm}$ in dark conditions. b Case 2, MRI showing left sided neck mass (arrow) in a child with intermittent and variable features of Horner's syndrome.
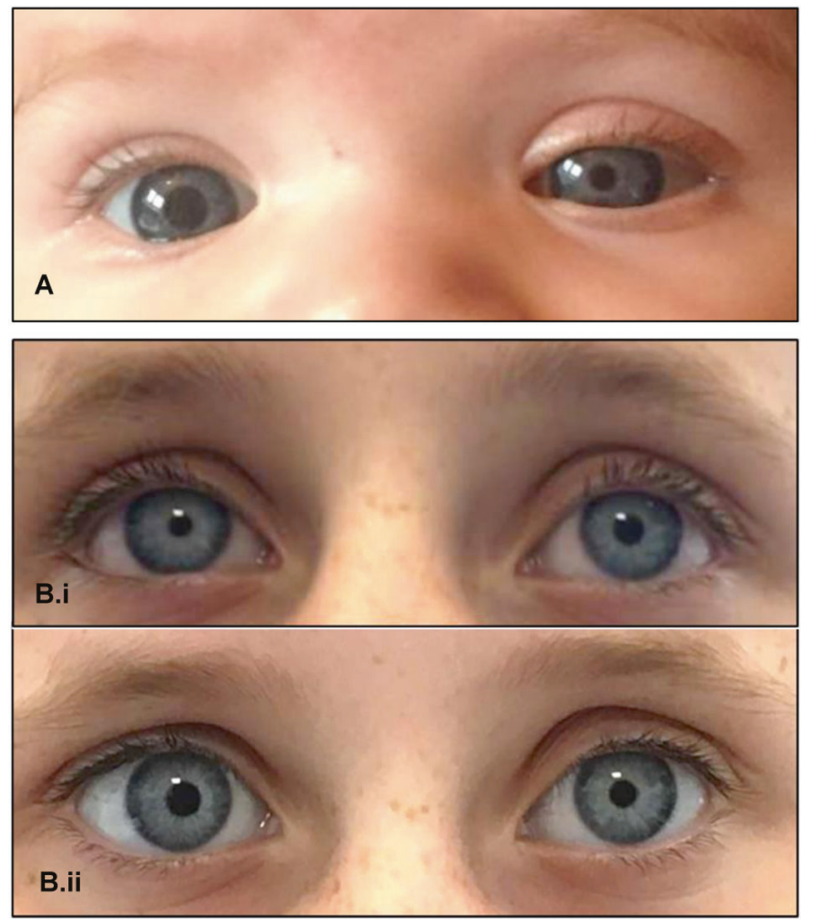

Fig. 3 Photographs capturing Horner's syndrome in two atypical cases. a Case 2; photo from the patient's parent capturing left HS in a child who examined completely normally when assessed in clinic. b (i) Case 3; pre apraclonidine $0.5 \%$ test showing right miosis but no appreciable ptosis. b (ii) Case 3; $60 \mathrm{~min}$ post apraclonidine $0.5 \%$ showing reversal of anisocoria. There is reversal of subtle right lower lid inverse ptosis also.

(right) and $4 \mathrm{~mm}$ (left) in light conditions; in dark they measured $5 \mathrm{~mm}$ (right) and $7 \mathrm{~mm}$ (left). There was no ptosis or heterochromia. The parents had not noted any ptosis but a teacher had commented that the child's right eyelid had been "droopy" on occasions. An Iopidine $0.5 \%$ test was carried out which unexpectedly caused reversal of anisocoria and reversal of subtle right lower lid inverse ptosis also (Fig. 3b), indicating HS. Urgent neuroimaging 
(MRI head, neck, thorax, and abdomen and USS pelvis) and urinary catecholamines tests were arranged but no cause of this acquired HS has been identified. As a side note, the child was reviewed $60 \mathrm{~min}$ after instillation of apraclonidine at which time the reversal of anisocoria was very apparent, but according to her parents it took at least 50 min before the apraclonidine, had this effect. In light of this event, it is now our practice to reassess pupils $1 \mathrm{~h}$ after instillation of apraclonidine in order to reduce the potential chance of missing a positive test.

\section{Case 4}

An 8-week-old baby was referred with a left ptosis noted since birth which measured $2-3 \mathrm{~mm}$ on initial ophthalmic assessment, with equal pupils in light and dark and otherwise normal examination. Birth history was unremarkable with a normal delivery and diagnosis of congenital ptosis was suspected. The child was reviewed at 6 months, by which time he had developed a miosed pupil on the left side which had first been noted by the parents 2 months previously. No pharmacological tests were done as there was no cocaine available and the child was considered too young for apraclonidine. MRI scan of head, thorax and abdomen was arranged but no cause of the HS was seen. On review, 1 year later, mild heterochromia was also noted with the left iris being lighter in colour.

\section{Discussion}

This study indicates a higher incidence of paediatric HS compared with previous published data. According to migration statistics, immigration in Northern Ireland is greater than emigration making our population ideal for epidemiological studies. The catchment area for the regional paediatric ophthalmology centre in Belfast encompasses most of NI for which the population of under 16 year olds in 2018 was 393,500 [ref. 3]. Therefore, the incidence of HS in this 6.5 year period was at least 2.54 per 100,000-contrasting with a population-based study in Minnesota who calculated an incidence of paediatric HS cases of 1.42 per 100,000 over a 40 year period [4].

Ten out of the $61(16.4 \%)$ children with anisocoria in our study period were diagnosed with HS, two of whom were infants with neuroblastoma. Another recent study found a positive cocaine drop test in as many as $12 / 35(31.4 \%)$ of children presenting with anisocoria [5], although the authors consider some of these may have represented physiological anisocoria with false-positive tests. The actual proportion of HS in our cohort may be higher due to potentially missed diagnoses-several cases which were labelled "physiological anisocoria" in our study population were not subjected to pharmacological tests to exclude HS, thus raising the possibility of undetected cases.

The rate of neuroblastoma in those with HS in this study was $20 \%$. A recent systematic review looking at paediatric HS found $12 / 152(7.9 \%)$ were a presenting sign of neuroblastoma [6]. Their conclusions affirmed those of previous authors [7] who indicate that neuroimaging is always warranted in paediatric HS with unknown aetiology. Our data would support this view in contrast to prior publications which have suggested HS is very rarely associated with neuroblastoma and that routine imaging in isolated infantile HS is not necessarily required [4, 8].

The proportion of HS cases which presented atypically in this series is particularly noteworthy. A previous publication has described three cases of atypical paediatric HS where anisocoria preceded development of ptosis, two of which were secondary to neuroblastoma [3]. The present study suggests that atypical HS where signs are intermittent or develop sequentially may represent as many as $40 \%$ of paediatric HS, and that these cases carry a fourfold increase risk of having underlying neuroblastoma compared with typical HS cases. It may be the very fact that neuroblastoma represents a dynamic process which leads to a greater chance of asynchronous presentations of HS in these cases.

Furthermore, we identified two HS cases which presented with ptosis and sequential development of ipsilateral miosis (cases 1 and 4). To the best of our knowledge these are the only cases of their kind reported in the literature and indicate the importance of considering HS as a differential in children with ptosis [9].

The majority of patients in this study (51/61) presented with just one symptom or sign and no other history suggestive of HS. Two of these were confirmed to have HS on further testing (albeit with no sinister cause identified), yielding a negative predictive value of $96.1 \%$ for HS in those with anisocoria or ptosis only at presentation. Therefore, from this data set, $\sim 4 \%$ of children with an apparently benign presentation of anisocoria or ptosis (and no other suggestive history) may be expected to have HS.

In view of these findings, we posit that all children presenting with anisocoria where the smaller pupil is deemed to be the abnormal one (or if any doubt) should have pharmacological tests to help exclude HS. There is lack of consensus regarding which pharmacological tests should be used amongst ophthalmologists, partly due to variable availability of cocaine eye drops in eye departments and concerns regarding side effects of apraclonidine in children. A few adverse events attributed to apraclonidine $0.5 \%$ (including lethargy, difficulty to rouse, bradycardia and low oxygen saturations) have been reported, but these have been in children less than 6 months old $[10,11]$. There is also important discussion regarding the relative efficacy of these pharmacological tests. 


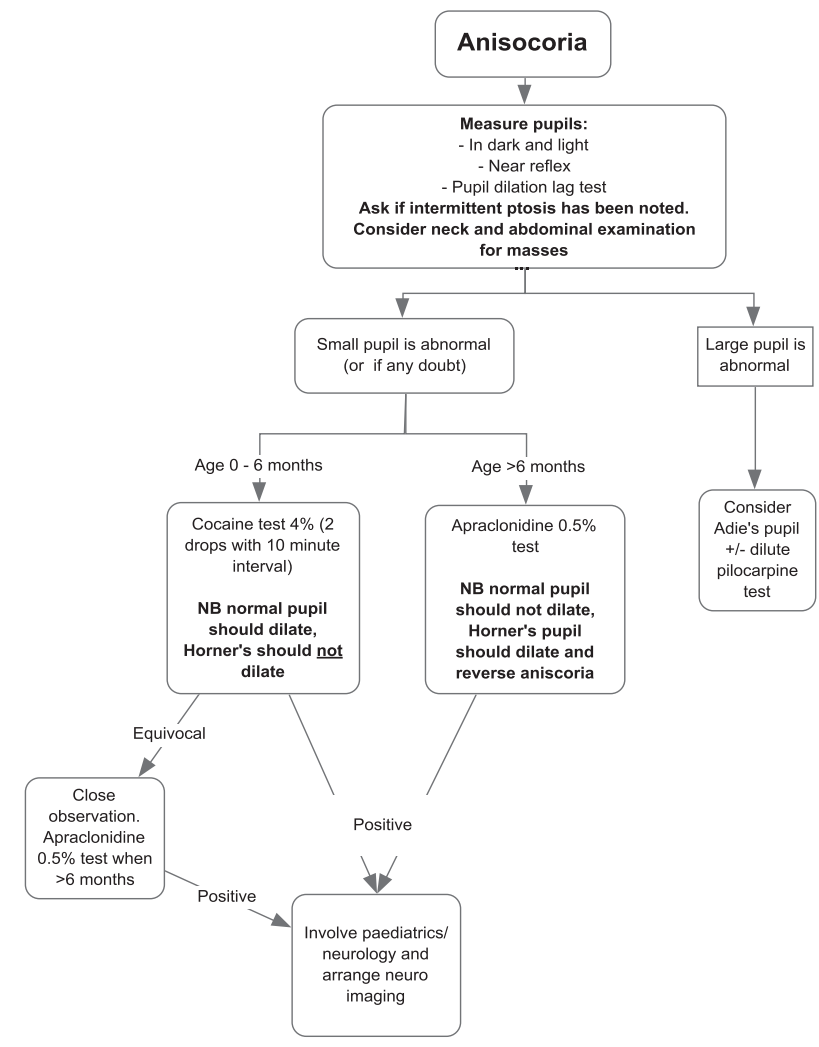

Fig. 4 Investigation algorithm for paediatric patients presenting with anisocoria.

An interesting recent study compared the sensitivity of cocaine $4 \%$ with apraclonidine $0.5 \%$ for detecting HS and reported that cocaine had a sensitivity of only $40 \%$ compared with apraclonidine which carried a sensitivity of 93\%, and therefore conclude that apraclonidine should be the gold standard pharmacological test for HS [12]. In this study, one drop of cocaine $4 \%$ was used and the pupils reassessed after a $40 \mathrm{~min}$ interval, whereas our practice is to use two drops of cocaine 4\% (10 min apart, to help ensure instillation of the drug in each eye) and re-assess in 60 min, which may produce a higher test sensitivity. The article also acknowledges there are some clinical scenarios, including in very young children, when cocaine is a more appropriate choice of test. Apraclonidine should also be used with caution in the acute phase of a possible HS as it takes 5-8 days for alpha- 1 receptors to up-regulate, and it may be appropriate to repeat the test after this period to reduce the risk of false negatives.

Taking all these things into consideration, we currently suggest that infants less than 6 months old with anisocoria should only be tested with cocaine eye drops even if the child needs to come back at a time when it is available. If the test result is equivocal, a follow up apraclonidine test when they reach 6 months may help confirm or exclude HS. For children from age 6 months, we advise apraclonidine $0.5 \%$ as the first line test (one drop in each eye and reassess after $60 \mathrm{~min}$. Patients younger than 2 years old should also be observed in clinic for at least $2 \mathrm{~h}$, and admitted to paediatrics if signs of lethargy, bradycardia or reduced respiratory rate develop). Pupil dilatation lag test and neck examination should also be performed. Figure 4 demonstrates a proposed investigation algorithm for children presenting with anisocoria. Braungart et al. [6] included a diagnostic algorithm for new onset paediatric HS in their recent systematic review which compliments the one presented here. For children with ptosis without miosis, the role of pharmacological testing is unclear. However, in such children it is important to consider HS as a differential diagnosis when assessing the child, and parents should be counselled to monitor for anisocoria and given access for urgent review if concerns arise.

Overall, the cases in this study highlight the importance of clinical vigilance when assessing children with anisocoria and/or ptosis. Pharmacological tests have an important role in the investigation of these cases and can help reassure parents (and clinicians) without necessitating neuroimaging (along with general anaesthetic) in many paediatric cases. Future studies with larger data sets may help elucidate further the prevalence of atypical HS and the usefulness and potential pitfalls of pharmacological testing of anisocoria in the paediatric population.

\section{Summary}

\section{What was known before}

- Paediatric HS can present atypically, including in cases of neuroblastoma.

- Paediatric HS can be a presenting sign of occult malignancy and warrants further investigation to exclude space occupying lesions.

- Pharmacological tests (apraclonidine $0.5-1 \%$ and cocaine 2-10\%) can help investigate possible HS; apraclonidine being the more sensitive but cocaine safer in infants.

\section{What this study adds}

- Atypical HS may occur in as many as $40 \%$ of paediatric HS cases and carry increased risk of significant underlying pathology.

- Atypical paediatric HS can include cases where ptosis precedes development of miosis.

- An algorithm to guide investigation of children presenting with anisocoria designed to reduce the risk of overlooking possible $\mathrm{HS}$ with its underlying cause. 


\section{Compliance with ethical standards}

Conflict of interest The authors declare that they have no conflict of interest.

Publisher's note Springer Nature remains neutral with regard to jurisdictional claims in published maps and institutional affiliations.

\section{References}

1. Jeffery AR, Ellis FJ, Repka MX, et al. Pediatric Horner syndrome. J AAPOS. 1998;2:159-67.

2. Pollard ZF, Greenberg MF, Bordenca M, Lange J. Atypical acquired pediatric horner syndrome. Arch Ophthalmol. 2010; 128:937-40.

3. Northern Ireland Statistics and Research Agency. 2016 Mid year population estimates for Northern Ireland. 2018. www.nisra. gov.uk.

4. Smith SJ, Diehl N, Leavitt JA, Mohney BG. Incidence of pediatric Horner syndrome and the risk of neuroblastoma: a populationbased study. Arch Ophthalmol. 2010;128:324-9.
5. Fierz FC, Gerth-Kahlert C. Long-term follow-up in children with anisocoria: cocaine test results and patient outcome. J Ophthalmol. 2017;2017:7575040.

6. Braungart S, Craigie RJ, Farrelly P, Losty PD. Paediatric Horner's syndrome: is investigation for underlying malignancy always required? Arch Dis Child. 2019;104:984-7.

7. Mahoney NR, Liu GT, Menacker SJ, Wilson MC, Hogarty MD, Maris JM. Pediatric Horner syndrome: etiologies and roles of imaging and urine studies to detect neuroblastoma and other responsible mass lesions. Am J Ophthalmol. 2006;142: $651-9$.

8. George NDL, Gonzalez G, Hoyt CS. Does Horner's syndrome in infancy require investigation? Br J Ophthalmol. 1998;82:51-4.

9. Pavone P, Cho SY, Praticò AD, Falsaperla R, Ruggieri M, Jin DK. Ptosis in childhood: a clinical sign of several disorders: case series reports and literature review. Medicine. 2018;97:e12124.

10. Watts P, Satterfield D, Lim MK. Adverse effects of apraclonidine used in the diagnosis of Horner syndrome in infants. JAAPOS. 2007;11:282-3.

11. Wright T, Freedman S. Exposure to topical apraclonidine in children with glaucoma. J Glaucoma. 2009;18:395-8.

12. Bremner F. Apraclonidine is better than cocaine for detection of Horner syndrome. Front Neurol. 2019;10:55. 